\title{
Las dificultades en la cuantificación de la violencia contra las mujeres en la pareja: análisis psicosocial $^{1}$
}

\section{The difficulties in the quantification of the violence against women in the couple: psychosocial analysis}

Victoria A. FERRER PÉREZ*
Esperanza BOSCH FIOL*
Teresa RIERA MADURELL*

Fecha de Recepción: 04-10-2004

Fecha de Aceptación: 30-10-2006

\section{RESUMEN}

De entre las diferentes formas de violencia contra las mujeres, la ejercida en el marco de la pareja o ex-pareja sentimental es la que alcanza tasas más elevadas, tanto a nivel mundial como en nuestro entorno. Sin embargo, determinar cuáles son esas tasas y, por tanto, arbitrar las medidas y recursos necesarios no es en absoluto tarea fácil. Por ello hemos considerado pertinente dedicar este trabajo al análisis las dificultades para cuantificar este problema.

A lo largo del texto se analizan los datos procedentes tanto de encuestas como de registros o denuncias y tanto de nuestro país como de otros.

Estos datos permiten, en primer lugar, concluir con bastante claridad que esta forma de violencia, así como su consecuencia fatal más irreversible, el femicidio, no es privativa de ningún país o conjunto de países concretos con unas determinadas características. Sin embargo, la magnitud concreta $y$, por tanto, las semejanzas y diferencias que pueda haber entre países y la evolución de este problema son temas mucho más dificiles de cuantificar. Se analizan y discuten estos resultados.

\footnotetext{
* Grupo de investigación "Estudios de género". Universidad de las Islas Baleares.

${ }^{1}$ Este trabajo se realizó en el marco de un proyecto de investigación financiado por el Programa Sectorial de Promoción General del Conocimiento de la Dirección General de Enseñanza Superior e Investigación Científica del Ministerio de Educación y Cultura (PB98-0122).
} 
Las dificultades en la cuantificación de la violencia contra las mujeres en la pareja: análisis social

\title{
PALABRAS CLAVE
}

Violencia contra las mujeres, Malos tratos, Estadísticas, Denuncias.

\begin{abstract}
Among the different forms of violence against women, domestic violence is the one that reaches higher valuations, so much World wide as in our environment. Nevertheless, to determine what are these valuations and, therefore, to arbitrate the measurements and necessary resources is not an easy task. For it we have considered necessary to dedicate this work to the analysis the difficulties to quantify this problem.

We analysed data of surveys and registrations from Spain and from different countries. These data permit in the first place, to conclude with plenty of clarity that this form of violence, as well as its more irreversible fatal consequence, the femicide, is not exclusive of any country or joint of countries with some characteristics. Nevertheless, the concrete magnitude and, therefore, the similarities and differences that can have between countries and the evolution of this problem are a lot more difficult themes to quantify. We analysed and discuss these results.
\end{abstract}

\section{KEY WORDS}

Violence against the women, Bad deals, Statistical, Accusations. 
La violencia contra las mujeres no es un fenómeno nuevo, pero algunos acontecimientos recientes y, muy especialmente, su inclusión en la agenda de organismos internacionales como la Organización de Naciones Unidas o la Organización Mundial de la Salud, han contribuido a visibilizarlo, pasando de considerarla un problema privado a un problema público (Bosch y Ferrer, 2000). Asi, uno de los motivos que han llevado a considerar este problema como prioritario por los organismos internacionales $\mathrm{y}$ a que éstos soliciten que los gobiernos del mundo le den ese mismo trato ha sido la constatación de que constituye un problema social y sanitario de primera magnitud, cuya extensión y consecuencias lo convierten en una prioridad de salud pública (Resoluciones $49.25 \mathrm{y}$ 50.19, OMS, 1998a).

De entre las diferentes formas de violencia contra las mujeres, la ejercida en el marco de la pareja o ex-pareja sentimental es una de las más frecuentes, tanto a nivel mundial como en nuestro entorno. Sin embargo, determinar cuáles son sus tasas exactas y, por tanto, arbitrar las medidas y recursos necesarios para afrontarla no es en absoluto tarea fácil. Como señalan Alberdi y Matas (2002), las dificultades para conocer las cifras de este problema son muy grandes, incluso en el caso de aquellas sociedades en las que ha aumentado la conciencia al respecto, y España no es una excepción, de modo que:

"nos encontramos con una gran escasez y una enorme diversidad de los datos que reflejan esta forma de violencia contra las mujeres (...) y apenas en los últimos años comienzan a realizarse registros de mujeres muertas o denuncias presentadas" (Alberdi y Matas, 2002, p. 115).

De hecho, la cuantificación de la vio- lencia contra las mujeres en la pareja ha sido y es motivo de importantes controversias, por lo que hemos considerado pertinente dedicar este trabajo a revisar el estado de esta cuestión.

\section{LA VIOLENCIA CONTRA LAS MUJERES EN LA PAREJA COMO DELITO OCULTO}

En relación a esta forma de violencia contra las mujeres (y al igual que ocurre en otros casos como las agresiones sexuales, el acoso, etc.) hay un acuerdo casi unánime en aceptar que se trata de un delito oculto, esto es, un delito que sale a la luz y se denuncia proporcionalmente muy poco y, por tanto, cuyas verdaderas cifras son dificiles de conocer (Abril, 1999; Caño, 1995; Echeburúa y Corral, 1998; Medina, 1994; Pérez del Campo, 1995; Sarasúa, Zubizarreta, Echeburúa y Corral, 1994; Zubizarreta, Sarasúa, Echeburúa, Corral, Sauca y Emperanza, 1994).

Las razones por las que las mujeres no denuncian la violencia que padecen a manos de sus parejas o ex-parejas sentimentales o no informan de su ocurrencia pueden ser muchas y variadas, y tanto de origen social como individual. Entre ellas estarian (Abril, 1999; Benitez, 1998; UNICEF, 2000; Watts y Zimmerman, 2002): El miedo a tener que continuar y/o reanudar la convivencia con su maltratador; el miedo, el desánimo o la falta de confianza en el sistema judicial o en los resultados que se pueden obtener al denunciar o hacer pública la violencia, o los elevados costes de emprender un proceso judicial; la dependencia económica (falta de recursos económicos, falta de vivienda, etc.) y/o afectiva de la víctima hacia el agresor; la no aceptación del fracaso de la relación de pareja; la consideración de que lo que ocu- 
rre en la pareja es un problema privado (y no un delito) que atañe sólo a la familia; los sentimientos de culpa 0 vergüenza, derivados de creer que ellas han provocado la violencia con su desobediencia, su fracaso o su infidelidad; la tolerancia social hacia el agresor $y / 0$ la falta de respuesta del entorno de la victima a este tipo de violencia; y las dificultades para que las propias victimas, el entorno (y a veces los/as profesionales) identifiquen como tal ciertas formas de violencia (sobre todo la psicológica y la sexual).

Pero, además de la reticencia 0 negativa a denunciar o hacer público el maltrato que se padece, hay también dificultades añadidas para conocer su magnitud derivadas del tipo de estadísticas que se realizan. Así, generalmente, se dispone sólo de informes sectoriales de ciertos organismos e instituciones que tratan de recoger o agrupar los datos sobre el tema; y, además, como veremos más adelante, suelen emplearse criterios distintos para recopilar datos, barajando definiciones diversas y obteniendo, por tanto, resultados dispares y dificilmente comparables (Goodman, Koss y Russo, 1993; OMS, 1998b).

Tratando de superar estas dificultades, los trabajos sobre el tema han combinado datos procedentes tanto de denuncias como de encuestas sobre violencia contra las mujeres en la pareja. A continuación analizaremos ambos tipos de datos, sus ventajas e inconvenientes y las cifras de las que disponemos en base a una y otra de estas fuentes, pero primero veremos cuáles son las recomendaciones de los organismos internacionales sobre la obtención y disponibilidad de datos sobre este problema (para poder analizar así hasta qué punto dichas recomendaciones se cumplen).

\section{LOS ORGANISMOS \\ INTERNACIONALES Y LOS DATOS SOBRE VIOLENCIA CONTRA LAS MUJERES EN LA PAREJA}

Diversos organismos internacionales vienen haciendo recomendaciones sobre la necesidad de obtener datos relativos a la violencia contra las mujeres en general, y en la pareja en particular, y sobre la forma más adecuada de hacerlo. Así, por ejemplo, la Plataforma para la Acción de la Declaración de Beijing, aprobada tras la IV Conferencia Mundial sobre las Mujeres (ONU, 1995) establecía en su objetivo estratégico D.2 la necesidad de estudiar las causas y consecuencias de la violencia contra la mujer y la eficacia de las medidas preventivas que se adopten. Para ello, y como acciones específicas, sugería recoger datos y elaborar estadísticas sobre la prevalencia de la violencia contra las mujeres, sus causas, naturaleza y consecuencias, así como sobre la eficacia de las medidas aplicadas, y divulgar ampliamente los resultados de dichas investigaciones al conjunto de la población.

En 1997 la Unión Europea estableció un mandato para que cada uno de sus países miembros recoja, elabore y publique anualmente datos sobre las diferentes formas de violencia contra las mujeres que ocurren en su territorio. Sin embargo, como ya remarcaron Alberdi y Matas (2002), este mandato se sigue sólo a medias y la recogida de datos no se está haciendo con criterios unificados, como se verá a continuación.

Posteriormente, los/as expertos/as del Consejo de Europa en las conferencias desarrolladas en 1999 y 2000 realizaron diversas propuestas para la recogida de información sobre el tema (DíazAguado, 2002, pp. 143-145): Realizar estadisticas desagregadas por género y desarrollar indicadores comunes para 
mejorar la valoración de la violencia contra las mujeres y evaluar la eficacia de las medidas adoptadas para prevenirla y paliar sus efectos; considerar ciertas influencias, frecuentemente olvidadas, que pueden alterar los resultados de encuestas e investigaciones como la posible variabilidad en función del contexto, del origen sociocultural o lingüístico o de los cambios que se están produciendo en los esquemas a partir de los cuales se conceptualiza el problema; avanzar en la estandarización y rigor de las encuestas tomando medidas como extraer muestras representativas de la población o controlar mejor los posibles errores de muestreo, establecer escalas con descripciones muy detalladas de los actos de violencia a partir de información directa de las víctimas, entrenar adecuadamente a los/as entrevistadores/as 0 recurrir a especialistas en lenguaje para evitar problemas de traducción; llevar a cabo encuestas e investigaciones sobre temas de interés prioritario incluyendo costes, causas y consecuencias de la violencia contra las mujeres; favorecer la cooperación entre las diferentes instancias públicas y privadas que trabajan el tema y promover la investigación tanto en redes nacionales como internacionales; establecer instituciones gubernamentales que coordinen la evaluación eficaz en la lucha contra el tema y consulten con los agentes sociales implicados; compilar y elaborar los resultados de las investigaciones para facilitar su divulgación al conjunto de la población. En esta linea, se sitúan las Recomendaciones aprobadas por la asamblea parlamentaria del Consejo de Europa, que ratificó el informe sobre violencia contra las mujeres en la pareja presentado por Olga Keltosova (2002).

Por su parte, el Observatorio de la Violencia del Lobby Europeo de Mujeres ${ }^{2}$ se constituyó con la idea de evaluar las politicas (tanto públicas, propuestas por los gobiernos y concretadas en planes de acción, como aquellas que llevan a cabo las ONGs) desarrolladas contra la violencia de género. Para lograr este objetivo se ha desarrollado una propuesta de indicadores relativos a diferentes cuestiones implicadas en el problema (actuaciones politicas, legislativas, educativas, ...), basadas en los indicadores propuestos por la Convención para la Eliminación de todas las formas de Violencia contra las Mujeres (CEDAW, n.d./2004). Por lo que se refiere a la recogida de datos, se recomienda elaborar: Estadísticas generales desagregadas por sexo; registros sistemáticos de todos los casos de violencia contra las mujeres; estadísticas anuales a este respecto y de evolución de los últimos 5 años; registros obligatorios por parte de la policía de todas las intervenciones relativas a casos de violencia; estadísticas integradas relativas al sistema de justicia penal; y, además, facilitar la participación de las estadísticas nacionales en la recogida de datos internacional; la colaboración con las ONGs; y la difusión de estadísticas y datos sobre la violencia a la sociedad en general.

El informe de Amnistía Internacional (2002) sobre la situación de la violencia de género en España se refiere también a esta cuestión cuando recomienda:

"Las autoridades deben recabar información estadistica actualizada sobre el grado de extensión de la violencia de género en el ámbito familiar. Estos datos deben hacerse públicos y difundirse ampliamente. Se deben suministrar estadisticas desagregadas que permitan diferenciar los actos de violencia de género cometidos contra las mujeres por parte de sus parejas o ex-

\footnotetext{
${ }^{2}$ Dirección de internet: http://www.observatorioviolencia.org
} 
parejas de otras formas de violencia" (p. 69).

Vemos pues que todas estas recomendaciones, y tanto las de organismos públicos como las de las ONGs, coinciden en señalar la necesidad de desarrollar mecanismos fiables y válidos para evaluar adecuadamente la magnitud de la violencia contra las mujeres en la pareja. Algunas de estas recomendaciones son muy recientes, pero otras, como las emanadas de la Conferencia de Beijing, tienen ya más de 10 años. En estos años y, al menos en el caso de España, la situación parece haber mejorado, pero aún quedan muchos pasos por dar. En los próximos apartados comentaremos los datos disponibles sobre esta cuestión.

\section{LOS DATOS SOBRE DENUNCIAS}

En primer lugar, analizaremos los datos procedentes de denuncias presentadas, de registros oficiales, de centros de atención a mujeres, etc. Su principal ventaja es que recogen información fehaciente, es decir, información de hechos acontecidos por lo que ofrecen resultados muy fiables (DíazAguado, 2002). Por contra, sus principales inconvenientes radican en que sólo tienen en cuenta los casos en los que las mujeres denuncian o solicitan ayuda, invisibilizando al resto; además, en algunos países la violencia contra la mujer en la pareja (en su totalidad o en algunos de sus aspectos) no está considerada como delito por lo que no aparece en los registros policiales; y, generalmente, recogen una cantidad de información muy limitada sobre las víctimas y sus características (Díaz-Aguado, 2002; Keltosova, 2002). Por tanto, podría decirse que estas fuentes ofrecen resultados que, aunque muy fiables, son incompletos.

\section{LOS DATOS SOBRE DENUNCIAS EN ESPAÑA.}

Los datos procedentes de registros oficiales que suelen emplearse en España son aquellos relativos a denuncias. En este sentido, cabe recordar que la Dirección General de la Policía comenzó a llevar una estadística sobre denuncias de malos tratos a mujeres a partir de 1984; la violencia fisica en el ámbito de la familia comenzó a ser considerada delito en 1989; hasta 1990 no comenzaron a aparecer datos estadísticos sobre el tema en las Memorias Anuales del Ministerio del Interior; y, finalmente, en 1992 se empezaron a analizar sistemáticamente dichos datos (Acale, 1999).

Por otra parte, y dados los diferentes modos en los que esta forma de violencia se ha tipificado en el ordenamiento juridico español, ha sido frecuente, como ya señaló Blanca Vázquez (1993), que su ocurrencia se hallara en las estadísticas judiciales dispersa entre los delitos de malos tratos, propiamente dicho, y otros apartados (otros delitos, faltas, delitos y faltas contra las personas, etc.) y también que las cifras oficiales no incluyeran las denuncias presentadas ante las policías autonómicas o en ciertos ámbitos geográficos.

A continuación, y a modo de ejemplo, presentamos la información sobre denuncias por violencia contra las mujeres en la pareja para el conjunto de España desde que éstas se recopilan, teniendo en cuenta que una misma persona puede haber hecho más de una denuncia, incluso en el mismo año (Ver Tabla 1).

Como puede verse, estos datos muestran unas cifras de denuncias muy similares (con ligeras oscilaciones) entre 1984 y 1995, y, a partir de entonces, un 


\section{Tabla 1. Registro de casos de violencia contra las mujeres en la pareja en España} (denuncias de delitos + faltas). Por años.

\begin{tabular}{|l|c|}
\hline$\Lambda \bar{n}$ o & Número de denuncias \\
\hline 1983 & 11.516 \\
\hline 1984 & 16.441 \\
\hline 1985 & 16.810 \\
\hline 1986 & 17.056 \\
\hline 1987 & 15.196 \\
\hline 1988 & 14.461 \\
\hline 1989 & 17.738 \\
\hline 1990 & 16.089 \\
\hline 1991 & 16.946 \\
\hline 1992 & 16.520 \\
\hline 1993 & 15.908 \\
\hline 1994 & 16.284 \\
\hline 1995 & 16.122 \\
\hline 1996 & 16.378 \\
\hline 1997 & 17.488 \\
\hline 1998 & 19.535 \\
\hline 1999 & 21.680 \\
\hline 2000 & 22.407 \\
\hline 2001 & 24.158 \\
\hline 2002 & 43.313 \\
\hline 2003 & 50.090 \\
\hline 2004 & 57.577 \\
\hline 2005 & 59.758 \\
\hline
\end{tabular}

Fuente: Datos elaborados por el Instituto de la Mujer (disponibles en su página web') a partir de las informaciones proporcionadas por cl Ministerio del Intcrior.

Criterios de recogida de estos datos: Hasta 1996 (inclusive) se refieren a denuncias de las esposas debido a los malos tratos recibidos de sus maridos; entre 1997 y 2001 (ambos inclusive) se relicren a denuncias de las mujeres debido a los malos fratos recibidos de sus cónyuges o análogos (incluycndo parcjas de hecho); a paríir de 2002 (inclusive) se refieren a denumcias de las mujeres debido a los malos tratos recibidos de sus cónyuges o análogos (incluyendo ex cónyuges, compañero sentimental, ex compañero sentimental, novio o ex novio).

${ }^{1}$ Direceión de intemel: htlp://www.mLas.cs/mujer/

incremento, ligero al inicio, pero constante, excepto en los últimos años, de modo que entre 2001 y 2002 la cifra prácticamente se duplica, lo cual podría estar relacionado, entre otras cosas, con los cambios de criterio al recogerlos (pasando de un criterio muy excluyente hasta llegar a otro más inclusivo).

Tomando como base el Censo de Población y Viviendas elaborado por el Instituto Nacional de Estadística español, en 1991 había en España 19.835.822 mujeres, pudiendo decirse que la tasa era en ese momento de 854 denuncias por violencia en la pareja por cada millón de mujeres. De acuerdo con el censo de 2001 el número de mujeres ascendió en nuestro país a 20.834.489, con una tasa de denuncias para ese año de 1.160 por cada millón de mujeres. Dado que éste sigue siendo el Censo vigente, podemos emplearlo para calcular la tasa actual que se situaría en 2.868 denuncias por cada millón de mujeres. Vemos, por tanto, que no sólo el número de denuncias ha aumentado, sino que la tasa de denuncias por violen- 
cia en la pareja por cada millón de mujeres se ha triplicado en nuestro país en los últimos 15 años.

En cuanto a la relación entre denuncias interpuestas y casos reales, los primeros textos que se publicaron en nuestro país sobre el tema (Caño, 1995; Medina, 1994; Pérez del Campo, 1995; Sarasúa et al., 1994; Zubizarreta et al., 1994) sugerian a título orientativo que, en general, el número de denuncias en España se correspondería, aproximadamente, a un 5\%-30\% de los casos de violencia contra las mujeres en la pareja existentes. En apoyo de las estimaciones más optimistas iría el hecho de que la Federación de Asociaciones de Mujeres Separadas y Divorciadas observó que del total de mujeres que solicitaron ayuda en sus centros en 2000, un 31\% llegaron a interponer después una denuncia, o que la Comisión para la Investigación de los Malos Tratos observó que el porcentaje ascendía a un $40 \%$ de las mujeres que solicitaban información (Lasheras y Pires, 2003). Sin embargo, como veremos más adelante, las encuestas sugieren que estas estimaciones se habrian quedado cortas.

\section{LOS DATOS PROCEDENTES DE DENUNCIAS O REGISTROS EN EL MUNDO}

En un reciente informe del Centro Reina Sofia para el Estudio de la Violencia (CRSEV) (Sanmartín, Molina y García, 2003), se presentan, entre otros, datos sobre denuncias o procedentes de registros en diferentes países del mundo. Para obtener esta información, el CRSEV contactó con un total de 95 países pero tan sólo la obtuvo para una decena de ellos como puede verse en la Tabla 2.

Cabe destacar que mientras en algunos casos (Mali, Mozambique, ...) los datos procedian de registros realizados por ONGs, en otros casos procedian de registros oficiales (como por ejemplo el dato de España) y aún en otros se informaba de que las fuentes habian sido de ambos tipos (como en el caso de USA, Japón, Alemania, etc.). Igualmente, no se indicaba si los datos proporcionados hacian referencia al conjunto del país o a una zona geográfica concreta ni cuáles habían sido los criterios empleados en su recogida (si incluían sólo pareja de derecho o también de hecho, si incluian

Tabla 2. Registro de casos de violencia contra las mujeres en la pareja en el mundo

\begin{tabular}{|l|c|c|}
\hline País & $\begin{array}{c}\text { Número de casos } \\
\text { en } 2000\end{array}$ & $\begin{array}{c}\text { Número de casos } \\
\text { por } 10.000 \text { mujeres } \\
\text { mayores de } 14 \text { años } \\
\text { en 2000 } \\
\text { Prevalencia }\end{array}$ \\
\hline Puerto Rico & 17.520 & 113,32 \\
\hline USA & 518.500 & 46,29 \\
\hline España & 22.407 & 12,71 \\
\hline Zimbawe & 1.748 & 5,04 \\
\hline Hungría & 1.017 & 2,31 \\
\hline Alemania & 6.488 & 1,81 \\
\hline Rumanía & 1.017 & 1,08 \\
\hline Mali & 270 & 0,87 \\
\hline Mozambique & 402 & 0,73 \\
\hline Japón & 962 & 0,17 \\
\hline
\end{tabular}

Fuente: Centro Reina Sofía para el Estudio de la Violencia (Sanmartín et al., 2003, p. 52). 
o no ex-parejas, etc.). Por tanto, y a pesar del evidente valor orientativo que puedan tener, sin mayores detalles sobre el modo en que fueron obtenidas, estas cifras deben ser manejadas con mucha prudencia ya que probablemente estén explicando más sobre el modo de recolectar la información y sus deficiencias que sobre el problema en sí mismo.

\section{LOS DATOS PROCEDENTES DE ENCUESTAS}

Al igual que ocurre con las denuncias y registros, también las encuestas sobre población general ayudan, directa o indirectamente, a comprender la magnitud de este problema y tienen ventajas e inconvenientes. Entre las ventajas cabe citar, por ejemplo (Diaz-Aguado, 2002) que incluyen más información puesto que acceden tanto a casos denunciados y/o públicos, como a aquellos que no lo son; que pueden repetirse en diferentes momentos y lugares, lo cual permite establecer comparaciones; y que permiten obtener una mayor cantidad de datos sobre las victimas, las circunstancias en las que ocurrió el suceso, sus efectos, etc. Entre sus posibles inconvenientes estarian los siguientes (Diaz-Aguado, 2002; Watts y Zimmerman, 2002): no siempre se emplean definiciones de violencia iguales o comparables entre sí; la población estudiada no siempre es similar (puede variar la edad, el estado civil, ...); pueden cometerse errores de muestreo, incluyendo la posibilidad de que se produzca la exclusión de quienes no se hallan en el marco muestral (por ejemplo, personas en riesgo de exclusión social); y pueden generar problemas ligados al recuerdo de las personas entrevistadas.

De hecho, la mayoría de datos disponibles sobre violencia contra las mujeres en la pareja provienen de encuestas, y las particularidades metodológicas de los diferentes estudios (definición de violencia empleada, tamaño de las muestras, método para su elección, tipo de preguntas formuladas, duración del estudio, ...) condicionan en gran medida el tipo y fiabilidad de los resultados obtenidos (UNICEF, 2000; Watts y Zimmerman, 2002).

\section{LOS DATOS PROCEDENTES DE ENCUESTAS EN ESPAÑA}

Por lo que se refiere a nuestro país, disponemos de algunos datos indirectos que dan una idea de la extensión del problema. Así, por ejemplo, en una encuesta realizada en 1990 por el Centro de Investigaciones Sociológicas (CIS) sobre población general (Cruz y Cobo, 1991) un 29\% de las personas entrevistadas afirmaban tener conocimiento personal de malos tratos ejercidos sobre mujeres y este porcentaje aumentaba hasta casi el $40 \%$ cuando las entrevistadas eran mujeres de 18 a 29 años. En otra encuesta sobre el tema (CIS, 2001) se observaron sólo ligeras variaciones, de modo que un $22 \%$ de las personas encuestadas decía conocer algún caso.

En cuanto a información directa sobre la ocurrencia de este delito, en un estudio realizado por Cáritas en la Comunidad de Madrid en 1989 en el que se entrevistó a mujeres casadas o con pareja estable, un $2 \%$ de ellas reconoció que su marido/pareja las maltrataba y un $7 \%$ se reservó su opinión sobre este tema. Por su parte, $\mathrm{M}^{\mathrm{a}}$ José Benitez (1998) realizó una encuesta de victimización observado que el 15\% de las mujeres entrevistadas había padecido malos tratos alguna vez y el 8\% los había padecido en los 5 años previos a la entrevista, pero sólo el $8 \%$ de víctimas había presentado denuncia. 
En 1999 el Instituto de la Mujer realizó una macroencuesta sobre ocurrencia de maltrato a más de 20.000 mujeres españolas mayores de edad (Alberdi y Matas, 2002; Instituto de la Mujer, 2000; Vives, 2001). En esta encuesta se preguntaba directamente a las mujeres si se sentían maltratadas en su relación de pareja, pero, además, se obtenían datos sobre los comportamientos vividos en dicha relación que permitían establecer la existencia de "situaciones objetivas de violencia", esto es, situaciones en las que, aunque las mujeres no tuvieran conciencia de ello, estaban en una posición de inferioridad con respecto de su marido o pareja, que se permitía tratarlas despreciativamente y que consideraba que podía imponerles su conducta y restringirles sus libertades. En estos casos se hablaba de la existencia de mujeres "técnicamente maltratadas".

Los datos obtenidos indicaron que el $42 \%$ de las entrevistadas (lo que traducido a la población española de mujeres mayores de edad serían 640.000 mujeres) admitía haber sufrido alguna forma de violencia de su entorno más cercano en el último año y, de ellas, el 52\% había sido maltratada por su pareja o ex-pareja (lo que traslado a la población general serían 368.000 mujeres). Es decir, el $2 ' 18 \%$ de mujeres mayores de 18 años del conjunto de la población española se sentía maltratada en su relación de pareja. Por otra parte, el 12'4\% de las entrevistadas (alrededor de 2.100.000 mujeres en la población general) estaba en una situación objetiva de violencia y, de ellas, el $742 \%$ sufría esa violencia a manos de su pareja o ex-pareja (1.550.000 mujeres). Es decir, el 9'2\% de mujeres mayores de 18 años del conjunto de la población española era "técnicamente maltratada" en su relación de pareja.

Como es fácilmente constatable, estas cifras se alejan de las de denuncias mos- trando que, efectivamente las denuncias representarían tan sólo un pequeño porcentaje de los casos existentes (entre menos del $2 \%$ y el $16 \%$, dependiendo del censo que tomemos como referencia y de si se trata de maltrato declarado o no).

\section{LOS DATOS PROCEDENTES DE ENCUESTAS EN EL MUNDO}

En la Tabla 3 se presenta un resumen de datos sobre violencia contra las mujeres en la pareja procedentes de encuestas realizadas en diversos países, elaborado para un trabajo anterior (Bosch y Ferrer, 2002) a partir de diferentes informes.

Resumiendo los datos presentados puede decirse que, entre un $10 \%$ y un $60 \%$ de las mujeres habrian padecido al menos un episodio de violencia en la pareja a lo largo de su vida y entre un $3 \%$ y un $54 \%$ habrian padecido violencia en la pareja a lo largo del año anterior a ser encuestadas, variando según países y tipos de violencia (física, psicológico y/o sexual).

Para interpretar adecuadamente estos datos es importante remarcar que, aunque todos proceden de encuestas, no son estrictamente comparables entre si ya que, tal y como se comentó anteriormente, las encuestas pueden haber sido realizadas con metodologías diversas. Así, los trabajos que se reseñan han sido recogidos a partir de muestras seleccionadas con criterios distintos, con distintas coberturas geográficas, con diferentes limites de edad y estado civil (casadas, viviendo en pareja, ...) y, sobre todo, a partir de definiciones y categorías disimiles para la medición y consideración de los diversos tipos de violencia, al tipo de preguntas realizadas, etc. (Heise y García-Moreno, 2003; UNICEF, 2000). 
Tabla 3. Información procedente de encuestas sobre la violencia contra las mujeres en la pareja en el mundo

\begin{tabular}{|c|c|c|}
\hline País & Año & $\begin{array}{l}\text { \% mujeres que han sufrido violencia a mamos de sus parcjas o } \\
\text { ex - parejas }\end{array}$ \\
\hline Alemania & $\begin{array}{l}\text { Sin } \\
\text { datos }\end{array}$ & $22 \%$ al menos un episodio de violencia. \\
\hline Australia & $1993-94$ & $22 \%$ violencia en el último año. \\
\hline Bangladesh & 1992 & $\begin{array}{l}47 \% \text { al menos un episodio de violencia; } 19 \% \text { violencia en el } \\
\text { último año. }\end{array}$ \\
\hline Bolivia & $1997-98$ & $\begin{array}{l}21 \% \text { violencia psicológica; } 17 \% \text { violencia fisica sin amenaza } \\
\text { para la vida; } 14 \% \text { violencia con amenaza para la vida. }\end{array}$ \\
\hline Camboya & 1996 & $16 \%$ violencia lisic:a; $8 \%$ lesiones. \\
\hline Cânadắ & 1993 & $\begin{array}{l}29 \% \text { al menos un episodio de violencia fisic:a; } 3 \% \text { violencia en cl } \\
\text { úlíimo ar̀o. }\end{array}$ \\
\hline China & 1999 & 10\% violencia cn cl último año. \\
\hline Colombia & $\begin{array}{l}1992 \\
1995\end{array}$ & $\begin{array}{l}20 \% \text { violencia física; } 33 \% \text { violencia psicológica; } 10 \% \text { violencia } \\
\text { sexual. } \\
19 \% \text { violencia física. }\end{array}$ \\
\hline Corea & 1992 & $38 \%$ violencia fisica en el ủltimo año. \\
\hline Costa Rica & $\begin{array}{l}1990 \\
1990 \\
1994\end{array}$ & $\begin{array}{l}54 \% \text { al menos un episodio de violencia. } \\
51 \% \text { violencia fisica varias veces al año: } 35 \% \text { regularmente. } \\
75 \% \text { violencia psicológica; } 10 \% \text { violencia fisica. }\end{array}$ \\
\hline Chilc: & $\begin{array}{l}1993 \\
1996 \\
1997 \\
2001\end{array}$ & 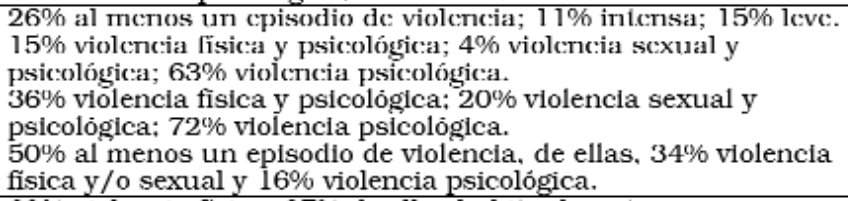 \\
\hline Ecuador & 1992 & $60 \%$ violencia fisica; $37 \%$ de ellas habitualmente. \\
\hline Egipto & 1996 & $35 \%$ al menos un episodio de violencia fisica. \\
\hline $\begin{array}{l}\text { Estados } \\
\text { Unidos }\end{array}$ & $\begin{array}{l}1983 \\
1986 \\
1991\end{array}$ & $\begin{array}{l}40 \% \text { violencia; } 22 \% \text { violencia en el último año; } 31 \% \text { violencia } \\
\text { lisic:a. } \\
28 \% \text { al monos un cpisodio de violencia fisic:a. } \\
39 \% \text { violencia; } 31 \% \text { violencia lisica; menos del } 12 \% \text { violencia } \\
\text { sexual. }\end{array}$ \\
\hline Etiopia & 1996 & $\begin{array}{l}45 \% \text { al menos un episodio de violencia; } 10 \% \text { violencia en el } \\
\text { último año. }\end{array}$ \\
\hline Filipinas & 1993 & $10 \%$ al menos un episodio de violencia. \\
\hline Finlandia & $\begin{array}{l}\text { Sin datos } \\
1998\end{array}$ & $\begin{array}{l}22 \% \text { al menos un episodio de violencia. } \\
40 \% \text { al menos un episodio de violencia. }\end{array}$ \\
\hline Francia & 1999 & $10 \%$ violencia habitual. \\
\hline Guatemala & 1990 & $74 \%$ violencia. \\
\hline Haití & 1996 & $36 \%$ al menos un episodio de violencia. \\
\hline India & $\begin{array}{l}1990 \\
1996\end{array}$ & $\begin{array}{l}75 \% \text { (de una casta) violencia frecuentemente. } \\
18-45 \% \text { (scgún distrito) de hombres c:asados cjercían violencia } \\
\text { lisica. }\end{array}$ \\
\hline Irlanda & $\begin{array}{l}\text { Sin } \\
\text { datos }\end{array}$ & $18 \%$ violencia. \\
\hline Israel & 1997 & $\begin{array}{l}32 \% \text { al menos un episodio de violencia física en el último año; } \\
30 \% \text { violencia sexual. }\end{array}$ \\
\hline Japón & 1993 & $59 \%$ al menos un episodio de violencia. \\
\hline Kenia & 1990 & $\begin{array}{l}42 \% \text { violencia fisica; de ellas, } 40 \% \text { regularmente y } 58 \% \\
\text { ocasionalmente. }\end{array}$ \\
\hline Malasia & 1993 & $39 \%$ violencia fisica en el último an̄o. \\
\hline México & $\begin{array}{l}1993 \\
1996 \\
1997\end{array}$ & $\begin{array}{l}57 \% \text { de mujeres urbanas y } 44 \% \text { de rurales violencia lisic:a. } \\
16 \% \text { violencia fisic:a. } \\
52 \% \text { al menos un episodio de violencia fisic:a; } 27 \% \text { violenceia en } \\
\text { el ültimo año. }\end{array}$ \\
\hline
\end{tabular}

Fuente: Elaboración propia para un trabajo anterior (Bosch y ferrer, 2002) a partir de

los informes de Ileise (1993). Eriksson (1997) o Kelly (2000) y de los dat.os proporcionados por la Organización Mundial de la Salud (lleise y Garcia-Moreno, 2003; OMS, 1998b) y UNICEF (2000). 
Tabla 3. Información procedente de encuestas sobre la violencia contra las mujeres en la pareja en el mundo (Continuación)

\begin{tabular}{|c|c|c|}
\hline País & Año & $\begin{array}{l}\text { \% mujeres que han sulfido violencia a manos de sus parcjas o } \\
\text { ex - parejas }\end{array}$ \\
\hline Nicaragua & $\begin{array}{l}1996 \\
1997 \\
1998\end{array}$ & $\begin{array}{l}52 \% \text { al menos un episodio de vlolencia física; } 27 \% \text { en el último } \\
\text { año; } 39 \% \text { violencia severa. } \\
46 \% \text { vlolencia psicologgica; } 28 \% \text { violencia fisica severa; } 30 \% \\
\text { violencia fisica moderada; } 18 \% \text { violencia sexual: } 53 \% \text { violencia } \\
\text { psicológica, fisica o sexual en el último año. } \\
28 \% \text { violencia fisica; } 10 \% \text { violencia sexual: } 29 \% \text { violencia fisica } \\
\text { y scxual. }\end{array}$ \\
\hline Nigcria & $1992-93$ & 31\% al menos un episodio de violencia. \\
\hline Norucya & 1989 & $25 \%$ al menos un cpisodio de violencia lisic:a o scxual. \\
\hline $\begin{array}{l}\text { Nucva } \\
\text { Zelanda }\end{array}$ & 1988 & $20 \%$ violencia lissic:a. \\
\hline Paises Bajos & 1992 & $21 \%$ al menos un episodio de violencia \\
\hline Palestina & 1998 & $54 \%$ violencia en el último año. \\
\hline Paraguay & $1995-96$ & $10 \%$ al menos un episodio de violencia. \\
\hline Perú & $\begin{array}{l}1997 \\
2000\end{array}$ & $\begin{array}{l}31 \% \text { violencia en el último año. } \\
41 \% \text { al menos un episodio de violencia: } 34 \% \text { episodios de } \\
\text { control; } 48 \% \text { humllaciones; } 25 \% \text { amenazas. }\end{array}$ \\
\hline Polonia & 1993 & $60 \%$ al menos un episodio de violencia; $25 \%$ violencia habitual. \\
\hline Port.ugal & 1995 & $52 \%$ al menos un cpisodio de violencia. \\
\hline Pucrio Rico & 1998 & $48 \%$ al menos un cpisodio de violencia. \\
\hline Rcino Unido & 1993 & $\begin{array}{l}30 \% \text { al menos un episodio de violencia lisica; } 11 \% \text { violencia cn } \\
\text { cl úlitimo año. }\end{array}$ \\
\hline $\begin{array}{l}\text { Repüblica } \\
\text { Moldava }\end{array}$ & 1997 & $\begin{array}{l}14 \% \text { al menos un episodio de vlolencia; } 7 \% \text { violencia en el } \\
\text { ültimo año. }\end{array}$ \\
\hline Sri Lanka & 1990 & $60 \%$ vlolencia. \\
\hline Sudäfrica & 1998 & $\begin{array}{l}27 \% \text { al menos un episodio de violencia; } 11 \% \text { violencia en el } \\
\text { último an̄o. }\end{array}$ \\
\hline Suiza & $\begin{array}{l}1994-96 \\
1997\end{array}$ & $\begin{array}{l}12 \% \text { al menos un episodio de violencia: } 6 \% \text { violencia en el } \\
\text { último año. } \\
20 \% \text { violcncia lisic:a. }\end{array}$ \\
\hline Tailandia & 1994 & $20 \%$ de hombres han cjercido violencia al menos una ve\%. \\
\hline T:anz:ania & 1990 & $60 \%$ al menos un cpisodio de violencia. \\
\hline Turquía & 1998 & $58 \%$ al menos un cpisodio de violencia. \\
\hline Uganda & 1996 & $\begin{array}{l}41 \% \text { violencia lisic:a; } 41 \% \text { de hombres han cjercido violencia } \\
\text { fisica. }\end{array}$ \\
\hline Zambia & 1992 & $40 \%$ al menos un episodio de violencia. \\
\hline Zimbawe & 1997 & $32 \%$ violencia fisica. \\
\hline
\end{tabular}

Fuente: Elaboración propia para un trabajo anterior (Bosch y Ferrer, 2002) a partir de

los informes de Heise (1993). Eriksson (1997) o Kelly (2000) y de los datos proporcionados por la Organización Mundial de la Salud (Heise y García-Moreno, 2003; OMS, 1998b) y UNICEF (2000).

En definitiva, a pesar de la disparidad de datos procedentes de las diferentes fuentes comentadas, lo que sí puede afirmarse, como ya apuntaron Heise y cols. (1999) o Walker (1999), es que ningún país del mundo está libre de esta forma de violencia.

\section{LOS FEMICIDIOS EN LA PAREJA}

El término femicidio es un neologismo que se ha impuesto recientemente para referirse a los asesinatos de mujeres (a manos de varones) como resultado extremo de la violencia de género que ocurre tanto en el ámbito privado como en el espacio público y que incluye tanto aquellas muertes de mujeres a manos de sus parejas o ex - parejas sentimentales, como a manos de familiares, agresores sexuales, etc.

En relación con esta cuestión, una 
primera puntualización a realizar es que no todos los países diferencian en sus estadísticas los homicidios de los femicidios y, en aquellos que sí lo hacen la distinción se ha introducido en la segunda mitad del siglo $X X$, salvo en el caso de Finlandia (que los diferencia desde 1754) (Sanmartin et al., 2003, p.46). Esto conlleva una ausencia de desagregación por género de las estadísticas sobre el tema que dificulta la extracción de conclusiones al respecto.

\section{LOS FEMICIDIOS EN LA PAREJA EN ESPAÑA}

En cuanto a las mujeres muertas a manos de sus parejas, en el estado español los datos oficiales los ha venido recogiendo el Instituto de la Mujer, a partir de las informaciones proporcionadas por el Ministerio del Interior. Estos datos tenían las siguientes características (Alberdi y Matas, 2002): Hasta 2002 sólo se incluia la información relativa a parejas que tuvieran un vínculo formal; la policía llevaba un registro diario de los delitos cometidos y el Ministerio del Interior se basaba en ese registro, incluyendo en sus estadísticas la identidad del acusado sólo cuando la policia lo hubie- ra identificado desde el primer momento; igualmente, en base a este registro, se incluian en las estadísticas sólo a aquellas mujeres victimas de violencia que fallecian inmediatamente (o en las primeras horas) tras la agresión; no se recogian aquellos sucesos atendidos por las policías autonómicas; los diferentes cuerpos de seguridad tenían métodos de registro propios y no unificados, lo cual podia hacer que los datos no siempre fueran estrictamente comparables; en algunos casos el Ministerio del Interior consideraba ciertos homicidios en el marco de la pareja como consecuencia de problemas económicos o similares, y no del maltrato.

Ante esta situación, diversas organizaciones feministas y en particular la Federación de Mujeres Separadas y Divorciadas comenzó en 1998 a llevar en su página de Internet ${ }^{3}$ un seguimiento con perspectiva de género del número de femicidios en la pareja a partir de la información aparecida en los medios de comunicación que no sólo se limitaba al día en que ocurría la agresión y que abarcaba todo el territorio nacional por lo que podian obtener datos más completos. En la tabla 4 puede verse la comparación entre ambos tipos de datos.

Tabla 4. Mujeres víctimas mortales de violencia en la pareja en España. Por años

\begin{tabular}{|l|c|c|c|}
\hline Año & $\begin{array}{c}\text { Datos Ministerio } \\
\text { del Interior }\end{array}$ & $\begin{array}{c}\text { Datos de Asociaciones } \\
\text { de Mujeres }\end{array}$ & $\begin{array}{c}\text { Datos del Instituto de } \\
\text { la Mujer corregidos }\end{array}$ \\
\hline 1995 & 65 & & \\
\hline 1996 & 97 & & \\
\hline 1997 & 33 & 38 & 54 \\
\hline 1998 & 34 & 52 & 53 \\
\hline 1999 & 45 & 61 & 54 \\
\hline 2000 & 43 & 60 & 71 \\
\hline 2001 & 46 & 65 & 72 \\
\hline 2002 & 52 & 72 & 62 \\
\hline 2003 & 68 & & \\
\hline 2004 & & & \\
\hline 2005 & & & \\
\hline
\end{tabular}

${ }^{3}$ Dirección de internet: http://www.separadasydivorciadas.org/ 
Estas disparidades suscitaron una fuerte critica social, especialmente por parte de las asociaciones de mujeres que acusaban a los organismos oficiales de propiciar una "guerra de cifras". Además, diversos trabajos de análisis, como el de Vives, Alvárez y Caballero (2003) ó el de la Fundación Mujeres (2003), se ocuparon de revisar estas disparidades, sugiriendo alternativas para corregirlas.

A la vista de todo ello, el Instituto de la Mujer decidió modificar sus criterios de recogida de datos sobre el tema y

"realizar su propia cuantificación, basada en un sistema mixto y unificado, en el que, partiendo de las noticias aparecidas en los medios de comunicación, que son utilizados como "sistema de alerta", cada uno de los casos, es, posteriormente, contrastado con los datos provenientes del Ministerio del Interior $y$, en un futuro, del ámbito judicial"”.

De acuerdo con este nuevo criterio, en la página web del Instituto de la Mujer se ofrecen datos sobre las mujeres muertas a manos de sus parejas o ex - parejas sentimentales a partir de 1999 que corrigen $y$, en muchos casos, amplian los que anteriormente proporcionaba esta institución, datos que se han incluido también en la Tabla 4. A partir de ellos, y tomando como referencia para su análisis el Censo de 2001, puede decirse que la tasa de mujeres muertas a manos de su pareja 0 ex-pareja por cada millón de mujeres ha oscilado en España entre 2'40 para el año 2001 y 3'46 para el año 2004.

\section{LOS FEMICIDIOS EN LA PAREJA EN EL MUNDO}

Por lo que se refiere a los femicidios a manos de la pareja o ex-pareja en otros países, el informe del CRSEV (Sanmartín et al., 20003) al que hemos hecho referencia anteriormente, ofrece los datos que se recogen en la Tabla 5.

De acuerdo con este informe, la pareja o ex-pareja sería responsable del $37 \%$ del total de femicidios que se cometen y del $70 \%$ de aquellos que se cometen en el ámbito familiar (Sanmartín et al., 2003).

En su momento, a la vista de estos datos se suscitó una importante polémica en nuestro país sobre el lugar que ocupaba España en este macabro "rảnking" y sobre el significado de que países con una larga tradición de lucha contra la discriminación de las mujeres y de implantación de políticas de igualdad (como los países nórdicos, por ejemplo) tuvieran tasas de femicidio en la pareja muy superiores a las de otros países sin este tipo de prácticas. Así, mientras algunos sectores creyeron ver ven esos datos el reflejo del fracaso de estas políticas, otros remarcaron la disparidad en el seguimiento del problema, desde la casi total ausencia en algunos casos hasta la gran precisión de datos en aquellos países más preocupados por estos problemas, que son también quienes más medidas igualitarias han impuesto.

Una última cuestión a comentar en torno al femicidio en la pareja tiene que ver con la relación entre muertes y denuncias. Aquí, como en casi todo lo que hace referencia a las diferentes formas de violencia de género, los datos son también discrepantes. Así, en el primer informe exhaustivo sobre el tema realizado en nuestro país, el del Defensor del Pueblo (1998) se estimaba en un 98\% el porcentaje de mujeres muertas a manos de su pareja o ex-pareja que previamente la habían denunciado por malos tratos.

${ }^{4}$ Dirección de internet: http://www.mtas.es/mujer/mujeres/cifras/violencia/muertes.htm 
Tabla 5. Mujeres víctimas mortales de violencia en la pareja en el mundo

\begin{tabular}{|c|c|c|}
\hline País & $\begin{array}{l}\text { No de casos } \\
\text { en } 2000 \\
\text { Incidencia }\end{array}$ & $\begin{array}{l}\mathrm{N}^{\circ} \text { de casos por millon de } \\
\text { mujeres de } 14 \text { ó más años } \\
\text { Prevalencia }\end{array}$ \\
\hline Rumanía & 119 & $12 ’ 62$ \\
\hline Estados Unidos & 974 & $8 ’ 70$ \\
\hline Finlandia & 19 & 8,65 \\
\hline Australia & 62 & $8 \cdot 15$ \\
\hline Noruega & 12 & $6 ’ 58$ \\
\hline Luxemburgo & 1 & 5’56 \\
\hline Dinamarca & 12 & $5 ’ 42$ \\
\hline Canadá & 67 & $5 ’ 21$ \\
\hline Suecia & 17 & 4’59 \\
\hline Reino Unido & 107 & $4 ’ 36$ \\
\hline Colombia & 63 & 4'31 \\
\hline Alemania & 128 & 3’58 \\
\hline España & 43 & 2,44 \\
\hline Japón & 134 & $2 \% 42$ \\
\hline Irlanda & 3 & $2 ’ 01$ \\
\hline Polonia & 30 & 1'85 \\
\hline Holanda & 12 & 1'83 \\
\hline Perú & 11 & $1 ' 26$ \\
\hline Zimbawe & 14 & 1'15 \\
\hline Islandia & 0 & 0 \\
\hline
\end{tabular}

Fuente: Centro Reina Sofía para el Estudio de la Violencia

(Sanmartín et al., 2003, p. 50).

Los datos que se han ido obteniendo posteriormente rebajan sustancialmente esa cifra, que el Observatorio contra la Violencia Doméstica y de Género del Consejo General del Poder Judicial cifró en el $19 \%$ en 2001 y en el $257 \%$ en 2002. En cuanto a las mujeres muertas a manos de su pareja o ex-pareja que estaban separadas y/o en proceso de separación de esa pareja, el Instituto de la Mujer maneja porcentajes que oscilan entre el 30 y el $45 \%$, según el año.

En su análisis sobre el tema, Vives y cols. (2003) no observaron para el estado español un patrón geográfico definido ni para las muertes ni para las denuncias $\mathrm{y}$, aunque observaron que en ciertas provincias se daban tasas elevadas, no obtuvieron correlación entre las provincias con mayores tasas de denuncias por violencia y aquellas con mayores tasas de femicidio a manos de la pareja, lo que les llevó a cuestionar que las denuncias fueran un factor relevante (ni protector, ni de riesgo) para morir a manos de la pareja sentimental.

En otros países los datos son variables. Así, según el informe del CRSEV (Sanmartín et al., 2003) el porcentaje de mujeres muertas a manos de su pareja o ex-pareja y que previamente había denunciado a esa pareja por malos tratos era del 56\% en Canadá en 1998 y del 30\% en Estados Unidos en 1997; y el porcentaje de mujeres muertas a manos de su pareja o ex-pareja y que estaban separadas y/o en proceso de separación de esa pareja era del 50\% en Estados Unidos en 1992, del 40\% en Australia en 1999, del 29\% en Dinamarca en 2000, del $275 \%$ en Canadá en 2002, del $17 \%$ en Chile en 2001 y del 15\% en Noruega en 2000 .

En relación con estas cuestiones, los/as responsables de algunas ONGs 
que han trabajado el tema resumen estas dificultades del modo siguiente: Ángeles Álvarez ${ }^{5}$ portavoz de la Red de Organizaciones Feministas contra la Violencia de Género, señala: "No creemos que mueran más mujeres en España que en otros paises de la UE, lo que sucede es que los indicadores que se utilizan para realizar los informes no siempre coinciden". Y el informe de Amnistía Internacional (2002) mencionado anteriormente ya apuntaba que:

"El ordenamiento penal sueco, además de los delitos de lesiones, amenazas, homicidio, etc., de carácter genérico, ha creado dos delitos autónomos: la violencia de hombres contra mujeres y la violencia contra menores. Quizás ésta sea una forma interesante de clarificar contra lo que se quiere luchar, identificando el aspecto central de la violencia de género, el sexo del autor y la víctima".

\section{¿QUUÉ ESTÁ PASANDO? HIPÓTESIS EXPLICATIVAS}

Datos como los presentados han suscitado un intenso debate internacional sobre el aumento de la violencia contra las mujeres en la pareja en los últimos años, dando origen a dos posibles hipótesis explicativas sobre sus causas: que las mujeres se muestren más dispuestas a denunciar estos problemas ("hipótesis benigna") 0 , por el contrario, que se esté produciendo un repunte de los casos de violencia de género ("hipótesis de la escalada”). Como ya señaló el informe del British Council (1999), probablemente ambas hipótesis sean ciertas.

En este sentido, la visibilización del problema, la mayor sensibilización social y la mayor receptividad de organizaciones e instituciones así como el mayor nivel de recursos públicos para protegerlas y ampararlas existente actualmente facilitaria que, efectivamente, hubiese más mujeres dispuestas a denunciar los problemas de violencia de género que padecen, confirmando en parte esta hipótesis "benigna" según la cual el maltrato ha existido siempre en la misma, o incluso en mayor, medida y lo que ocurre es que ahora se hace público.

Pero, por otra parte, existe otra posibilidad explicativa, bastante menos halagüeña. Así, si partimos de que el desequilibrio de poder entre géneros está en la base de este tipo de violencia (como se considera, por ejemplo, desde la perspectiva feminista de análisis), cabe pensar que los cambios en las relaciones de género y los avances en pro de las mujeres pueden hacer que, a corto plazo, aumente la violencia contra ellas dadas las dificultades de ciertos varones para adaptarse a estas nuevas situaciones y para acomodarse a las nuevas pautas en las relaciones de pareja. El aumento alarmante de las agresiones con intencionalidad de matar y de los asesinatos junto con aquellos casos en que los intentos de romper la relación de pareja llevan a un incremento de la intensidad de la violencia reforzarían esta hipótesis de la escalada, que complementaría la anterior.

\section{A MODO DE CONCLUSIÓN}

Como se ha mostrado a lo largo de este trabajo, los datos disponibles sobre violencia contra las mujeres en la pareja arrojan algunas luces, pero también muchas sombras sobre este problema. Estos datos permiten, en primer lugar, concluir que la ocurrencia de esta violencia, así como su consecuencia fatal más irreversible, el femicidio, no es pri-

\footnotetext{
${ }^{5}$ Declaraciones al diario "El País". 18 de julio de 2003.
} 
vativa de ningún país o conjunto de paises concretos con unas determinadas características. Y, éste, que parece un dato obvio, resulta de gran importancia para contrarrestar el mito de que la violencia contra las mujeres (en general y en la pareja) es privativa de sectores marginales, pobres e incultos, mito que aún está fuertemente arraigado en algunos sectores sociales o incluso profesionales y que es necesario desmentir.

Más allá de esta primera constatación, la siguiente conclusión que puede extraerse de todos estos datos es que la magnitud concreta $y$, por tanto, las semejanzas y diferencias que pueda haber entre países y la evolución de este problema son temas mucho más dificiles de cuantificar. Cabe por tanto reflexionar sobre los motivos para ello.

En este sentido, y como ya se ha comentado, una de las principales razones para esta dificultad tiene que ver con cuáles son los datos disponibles y cuáles sus características. Así por ejemplo, ya en el informe de CRSEV (Sanmartin et al., 2003), al que nos hemos referido en repetidas ocasiones, se resumen los principales problemas detectados en la recopilación de datos del modo siguiente: Muchas de las estadisticas que se realizan no están desagregadas por género; en ocasiones no se tiene en cuenta la relación que existe entre el agresor y la victima; las definiciones del problema estudiado que se manejan no coinciden plenamente entre los diferentes paises; no siempre se manejan adecuadamente los conceptos de incidencia y prevalencia; e, incluso, el concepto de población no siempre se usa en el mismo sentido (así, en ocasiones, la población de referencia son todas las mujeres de un país, en otras son las de una determinada edad, en otras las de un cierto estado civil, ...).
Además de todos estos inconvenientes citados en el propio informe, una lectura atenta del mismo desvela otro importante problema. Así, se especifica que:

"Para elaborar este apartado (Estadistica) el Centro Reina Sofia ha contado con la colaboración de las ONGs más representativas de los distintos países. Más tarde se han solicitado estadisticas de los Ministerios de Justicia, Interior y Asuntos Sociales (alli donde habia). Finalmente, se ha tomado contacto con las Direcciones Generales de la Policía" (p. 42).

Y, efectivamente se ofrece una completísima lista (pp. 43-44) indicando qué organismos de cada país han colaborado en la recogida de los datos. Sin embargo, y a pesar de esta minuciosidad, cuando se presentan los datos (sobre femicidios, registros, ...) no se especifica de qué fuente concreta procede cada uno (oficial u ONG), ni tampoco si había discrepancias entre las distintas fuentes y, en su caso, cómo se resolvieron éstas. Recordemos, a título de ejemplo, que en los femicidios cometidos en España existían importantes discrepancias entre los datos oficiales y los de las ONGs. y en otros países podría ocurrir algo similar, lo cual iría en detrimento de la fiabilidad de los datos, llegando a ponerlos en cuestión.

Por lo que se refiere a la obtención de los datos a partir de encuestas o registros oficiales y denuncias, como ya se ha comentado, ambos tienen ventajas pero también inconvenientes, por lo que parece adecuado considerarlos como métodos de recogida de datos complementarios y no excluyentes (Díaz-Aguado, 2002), asi como extremar el cuidado en su recolección.

En este sentido, y en cuanto a los registros oficiales, el informe de DíazAguado (2002) para la reunión del Con- 
sejo de Ministros de la Unión Europea revisó detalladamente las formas de registrar la violencia contra las mujeres en la pareja en los estados de la Unión Europea con sugerencias sobre los modelos a seguir, los datos que deben incluirse, etc. y que resultan recomendaciones particularmente adecuadas.

En cuanto a las encuestas, se han comentado ya algunas sugerencias para mejorarlas incluyendo (Díaz-Aguado, 2002): Aumentar la comparabilidad de los datos de los diferentes países, homogeneizando la forma de recogerlos en estudios comunes con preguntas consensuadas; incluir preguntas comunes en encuestas periódicas; incorporar sistemáticamente a las estadísticas nacionales e internacionales (como EUROSTAT) datos sobre violencia contra las mujeres; eliminar problemas de marco muestral encuestando también a mujeres de colectivos marginados; perfeccionar las preguntas y las formas de preguntar; incorporar a las preguntas el análisis de la exclusión social; desarrollar estadísticas desagregadas para poder analizar mejor la ocurrencia del problema por estratos socioeconómicos, etc. Todas ellas (junto con las descritas anteriormente) deberían ser adecuadamente tenidas en cuenta en la recolección de datos mediante encuestas.

En el caso de España muchas de esas recomendaciones ya han incorporado, como prueba la abundancia de datos, muchos de los cuales se han mostrado en este trabajo. Sin embargo, lo que sí sería imprescindible sería clarificar y unificar los criterios a partir de los cuáles se recogen los datos. Precisamente, la creación (RD253/2006 de 3 de marzo, BOE núm. 62 de 14-03-2006) del Observatorio Estatal de Violencia sobre la
Mujer, entre cuyas funciones están "Actuar como órgano de recogida, análisis y difusión de información periódica, homogénea y sistemática, relativa a la violencia de género (...). A tal efecto, se creará una base de datos de referencia y se normalizará un sistema de indicadores mediante el establecimiento de criterios de coordinación para homogeneizar la recogida y difusión de datos", parece ir encaminada a paliar todas 0 algunas de estas dificultades, unificando criterios y metodologias en la recogida de los datos sobre este problema.

Cabe reseñar que también organismos internacionales como la OMS están trabajando en esta cuestión. Concretamente, bajo la dirección de la Dra. Claudia García Moreno (OMS, 2001) . Se $^{2}$ está desarrollando desde 1997 el "Estudio multi-país sobre la salud de las mujeres y la violencia doméstica" que está recogiendo datos de 10 países (Bangladesh, Brasil, Japón, Namibia, Nueva Zelanda, Perú, Samoa, Serbia, Tanzania, Tailandia) empleando en todos ellos una misma metodologia (que combina elementos cuantitativos y cualitativos) para recoger información sobre la incidencia y prevalencia de la violencia contra las mujeres, de las estrategias que ellas emplean para hacerle frente, sus consecuencias, los factores determinantes y de riesgo, y así elaborar nuevos instrumentos para medir la violencia transculturalmente y desarrollar nuevas estrategias para la investigación con perspectiva y sensibilidad de género.

Por lo que se refiere a las hipótesis explicativas sobre la violencia contra las mujeres en la pareja, posiblemente, tal y como ya comentamos al presentarlas, tanto la hipótesis benigna como la de la

\footnotetext{
${ }^{6}$ En la página web de la Organización Mundial de la Salud (OMS) (http://www.who.int/mipfiles/2255/ FinalVAWprogressreportforwebpagewithoutcover.pdf) se ofrecen informes sobre los avances de este trabajo.
} 
escalada sean parcialmente ciertas y complementarias.

Así, y aplicándolas al caso de nuestro país, es indudable la importancia del trabajo de los organismos públicos (y especialmente del Instituto de la Mujer desde su creación en 1983 y de los organismos de igualdad autonómicos primero y municipales más recientemente) y de los grupos y organizaciones de mujeres. Ese trabajo ha contribuido a la sensibilización en torno al problema y ello tanto por parte de las mujeres que lo sufren (que serían así más capaces de identificar lo que les sucede, de catalogarlo como fuera de lo normal y de emprender acciones para hacerlo público y ponerle fin) como por parte de los/as profesionales que han de atender a esas mujeres (y que estarán pues mejor preparados para darles apoyo y soluciones). Todo ello facilitaría, como es lógico, la visibilización de los casos preexistentes, yendo en apoyo de la denominada "hipótesis benigna" y ayudándonos a comprender, al menos en parte, por qué en los países con mayores mecanismos de ayuda a las mujeres éstas podrían tener menos reparos a la hora de denunciar y/o hacer pública su relación violenta.

La hipótesis benigna, sin embargo, no explica porqué sigue sucediendo violencia contra las mujeres en la pareja en aquellas sociedades más igualitarias. Y para comprenderlo podríamos recurrir a la denominada "hipótesis de la escalada" o, como resume Angeles Álvarez"; "hay una reacción violenta por parte de los varones ante los espacios que están con- quistando las mujeres en el mundo. Y esa reacción está siendo mayor en países donde hay más espacios de libertad, pero no lo tenemos (aún) sustentado en ningún estudio".

En el caso concreto de España podríamos relacionar la evolución del maltrato con los avances legislativos de los últimos años y su más lenta asunción por parte de la sociedad. Debemos recordar que la actual Ley del Divorcio data de 1981. Durante el largo período de la dictadura el divorcio no sólo era imposible sino que la separación era considerada como un gravísimo atentado contra los cimientos mismos de la sociedad, que partía de premisas como la indisolubilidad del matrimonio o la obediencia debida de la mujer a su marido. Con el paso del tiempo el divorcio se ha ido convirtiendo en una posibilidad aceptada por la sociedad española y, por tanto, son más las mujeres que lo ven como una salida a la situación de insatisfacción o de violencia en la que viven, lo cual puede desencadenar una reacción de aumento extremo de la violencia en aquellos varones incapaces de aceptar esta ruptura o su posibilidad.

En definitiva, tanto una mejora en el registro de la violencia contra las mujeres en la pareja como una visión más amplia en su análisis (que contemple, entre otras cosas, las dos hipótesis explicativas a las que hemos hecho referencia) puede ayudarnos a realizar una radiografia más exacta de este problema que contribuya a poner los medios necesarios para hacerle frente.

\footnotetext{
${ }^{7}$ Declaraciones al diario “El Mundo”. Suplemento Crónica, núm. 407. 2003.
} 


\section{BIBLIOGRAFÍA}

Abril, C. (1999). Violencia doméstica: Planteamiento general. En J.D. Martín (Coord.), La violencia sobre la mujer en el grupo familiar. Tratamiento jurídico y psicosocial (pp. 21-32). Madrid: Colex.

Acale, M. (1999). El delito de malos tratos fisicos y psíquicos en el ámbito familiar. Valencia: Tirant lo Blanch.

Alberdi, I. y Matas, N. (2002). La violencia doméstica en España. Barcelona: Fundación La Caixa.

Amnistía Internacional (2002). No hay excusa. Violencia de género en el ámbito familiar y protec ción de los derechos humanos de las mujeres en España. [documento www] Disponible en: http://www.a-i.es (20 de noviembre de 2002).

Benítez, M.J. (1998). Violencia intrafamiliar: La mujer maltratada. Cuadernos de Derecho Judicial, 7, 273-293.

Bosch, E. y Ferrer, V.A. (2000). La violencia de género: De cuestión privada a problema social. Intervención Psicosocial, 9(1), 7-19.

Bosch, E. y Ferrer, V.A. (2002). La voz de las invisibles. Las víctimas de un mal amor que mata. Madrid: Cátedra. Colección Feminismos.

British Council (1999). La violencia contra las mujeres. Manchester: British Council.

Caño, X. (1995). Maltratadas. El infierno de la violencia sobre las mujeres. Madrid: Temas de Hoy.

Cáritas (1989). Los malos tratos en la familia. Las mujeres maltratadas. En Pobreza y desigual dad en la Comunidad de Madrid. Necesidades, recursos y balance social. Madrid: Popular.

CEDAW (Convención para la Eliminación de todas las formas de Violencia contra las Mujeres) (n.d./2004). Recomendaciones CEDAW. [documento www] Disponible en: http://www.observatorioviolencia.org/indicadores.asp (22 de julio de 2004)

CIS (Centro de Investigaciones Sociológicas) (2001). Avance provisional de resultados. Distribuciones marginales. Barómetro de marzo de 2001 (Estudio 2411 documento www). Disponible en: http//www.cis.es
Coker, A.L., Smith, P.H., McKeown, R.E. y King, M.J. (2000). Frequency and correlates of intimate partner violence by type: physical, sexual, and psychological battering. American Journal of Public Health, 901(4), 553-559.

Cruz, P. y Cobo, R. (1991). Las mujeres españolas: lo privado y lo público. Madrid: Centro de Investigaciones Sociológicas.

Defensor del Pueblo (1998). Violencia contra las Mujeres. Madrid: Oficina del Defensor del Pueblo.

Díaz-Aguado, M.J. (2002). Guía de buenas prácticas para paliar los efectos de la violencia contra las mujeres y conseguir su erradicación. Presidencia de la Unión Europea. Santiago de Compostela. [documento www] Disponible en: http://www. ue2002.es (22 de julio de 2004).

Echeburúa, E. y Corral, P. (1998). Introducción. En E. Echeburúa y P. Corral (Eds.), Manual de violencia familiar (pp. 1-8). Madrid: Siglo XXI.

Eriksson, M. (Ponente) (1997). Violencia contra las mujeres (A4-0250/97). [documento WWW]. Disponible en: http://nodo50.ix.apc.org/mujeres $\mathrm{red} /$ violencia-ue/htm.

Fundación Mujeres (2003). Informe sobre violencia contra las mujeres en España. Tasas y tendencias de homicidio/asesinato 1999/2003. [documento www] Disponible en: http://www.redfeminista. org/Noticia.asp?ID=849 (22 marzo 2004).

Goodman, L.A., Koss, M.P. y Russo, N.F. (1993). Violence against women: Physical and mental health effects. Part I. Research findings. Applied \& Preventive Psychology, 2, 79-89.

Heise, L.L. (1993). Violence against women: the hidden health burden. World Health Statistics Quarterly, 46(1), 78-85.

Heise, L.L., Ellsberg, M. y Gottemoeller, M. (1999). Para acabar con la violencia contra la mujer. Population Reports, 4. Serie L, número 11. Temas sobre salud mental.

Heise, L. y Garcia-Moreno, C. (2003). La violencia en la pareja. En E.C. Krug, L.L. Dahlberg, J.A. Mercy, A.B. Zwi y R. Lozano (Eds.), Informe Mundial sobre Violencia y Salud (pp. 96-131). Washington DC: OMS-OPS (Orig. 2002). 
Instituto de la Mujer (2000). La violencia contra las mujeres. Resultados de la macroencuesta. Madrid: Instituto de la Mujer.

Kelly, L. (2000). ¿Cómo en casa en ninguna parte? La violencia doméstica, la seguridad de las mujeres y los niños y la responsabilidad de los hombres. Carpeta de Documentos del Foro Mundical de Mujeres contra la Violencia (pp. 25-36). Valencia: Centro Reina sofia para el Estudio de la Violencia.

Keltosova, O. (2002). Domestic violence. Doc. 9525. Repor Committee on Equal Opportunities for Women and Men. Council of Europe. [documento www] Disponible en: http:/assembly.coe.int/ (26 de julio de 2004).

Lasheras, M.L. y Pires, M. (2003). La violencia contra las mujeres considerada como problema de salud pública. Madrid: Instituto de Salud Pública.

Medina, J. (1994). Perfil psicosocial y tratamiento del hombre violento con su pareja en el hogar. En E. Echeburúa (Eds.), Personalidades violentas (pp. 153-167). Madrid: Pirámide.

OMS (Organización Mundial de la Salud) (2001). WHO multi-country study on women's health and consequences. Progress report. [documento www] Disponible en: http://www.who.int/ gender/violence/multicountry/en/print.html (18 septiembre 2003)

OMS (Organización Mundial de la Salud). Salud Familiar y Reproductiva. OPS. División de Salud y Desarrollo (1998a). Violencia contra la mujer. Un tema de salud prioritario. Washington DC: OMS/OPS.

OMS (Organización Mundial de la Salud) (1998b). Violencia contra la mujer (documento www). Disponible en: http://www. who.int/frhwhd/VAW/infopack/Spanish/violenciainfopack.htm

ONU (Organización de Naciones Unidas) (1995). Declaración de Beijing. IV Conferencia Mundial sobre las Mujeres. A/CONF. 177/20.
Pérez del Campo, A.M. (1995). Una cuestión incomprendida. El maltrato a la mujer. Madrid: Horas y Horas.

Sanmartín, J., Molina, A. y García, I. (Eds.) (2003). Informe internacional 2003. Violencia contra la mujer en las relaciones de pareja. Estadisticas y legislación. Valencia: Centro Reina Sofía para el Estudio de la Violencia.

Sarasúa, B., Zubizarreta, I., Echeburúa, E. y Corral, P. (1994). Perfil psicológico del maltratador a la mujer en el hogar. En E. Echeburúa (Eds.) Personalidades violentas (pp. 111-128). Madrid: Pirámide.

UNICEF (2000). Informe Innocenti (documento www). Disponible en: http://www.unicef-icdc. org/publications/ pdf/digest6s.pdf

Vázquez, B. (1993). La violencia familiar y la agresión sexual como objeto de la pericial psicológica. En J. Urra y B. Vázquez (Comps.), Manual de psicología forense (pp. 273-318). Madrid: Siglo XXI.

Vives, I. (2001). Las políticas públicas desde la administración del estado. En R. Osborne (Coord.), La violencia contra las mujeres. Realidad social y politicas públicas (pp. 79-91). Madrid: UNED.

Vives, C., Alvárez, C. y Caballero, P. (2003). Violencia del compañero intimo en España. Gaceta Sanitaria, 17(4), 268-274.

Walker, L.E.A. (1999). Psychology and domestic violence around the world. American Psychologist, $54(1), 21-29$.

Watts, C. y Zimmerman, C. (2002). Violence against women: global scope magnitude. The Lancet, 359, 1232-1237.

Zubizarreta, I., Sarasua, B., Echeburúa, E. Corral, P., Sauca, D. y Emperanza, I. (1994). Consecuencias psicológicas del maltrato doméstico. En E. Echeburúa (Eds.), Personalidades violentas (pp. 29-152). Madrid: Pirámide. 